Figure 1. Rate of radiographic progression (RP) by MBDA category for patient groups based on concordance or discordance of Patient Global Assessment (PGA) and Evaluator Global Assessment (EGA).

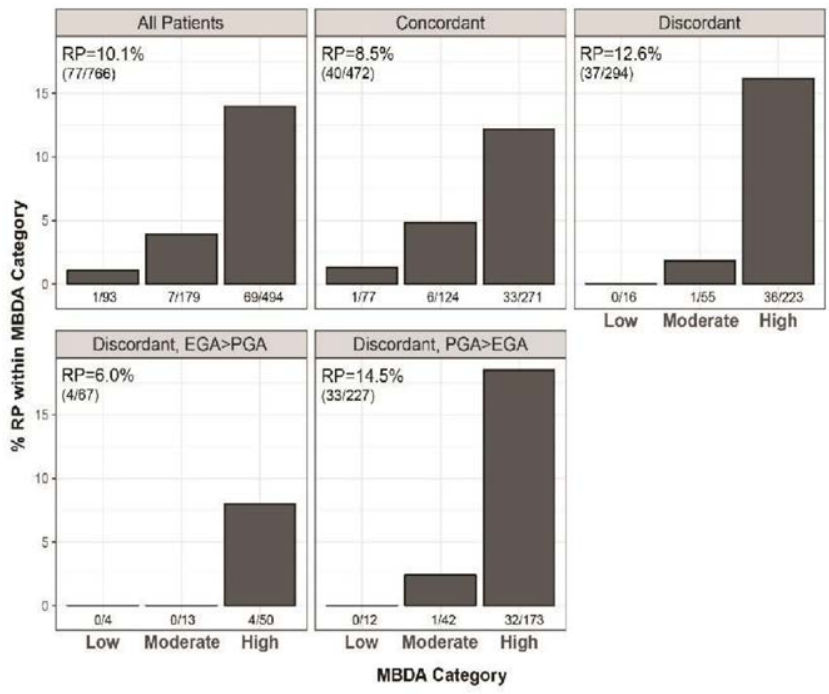

Disclosure of Interests: Leonard Calabrese Grant/research support from: AbbVie, Bristol-Myers Squibb, Cresecendo, Genentech, Gilead, GlaxoSmithKline, Horizon, Janssen, Novartis, and Sanofi., Michael E. Weinblatt Shareholder of: Canfite, Inmedix, Scipher, and Vorso, Consultant of: AbbVie, Aclaris, Amgen, Bayer, Bristol-Myers Squibb, Crescendo Bioscience, Corrona, EqRX, GSK,Genosco, Gilead, Lilly, Novartis, Pfizer, Roche, Set Point, Grant/research support from: Bristol-Myers Squibb, Myriad Genetics, Inc., Eli Lilly and Sanofi, Nancy Shadick Consultant of: BMS, Grant/research support from: Lilly, mallinckrodt, BMS, Amgen and Sanofi, Cecilie Heegaard Brahe: None declared, Mikkel Østergaard Consultant of: Abbvie, BMS, Boehringer-Ingelheim, Celgene, Eli-Lilly, Centocor, GSK, Hospira, Janssen, Merck, Novartis, Orion, Pfizer, Regeneron, Roche, Takeda, and UCB, Grant/research support from: AbbVie, BMS, Celgene, Myriad Genetics, Inc., Janssen, and Merck, Merete L. Hetland Speakers bureau: Orion, Grant/research support from: AbbVie, Biogen, BMS, CelltrionRoche, Myriad Genetics, Inc., Eli Lily, MSD, Pfizer, and UCB, Megan Horton Shareholder of: Myriad Genetics, Inc., Employee of: Myriad Genetics, Inc., Darl Flake Shareholder of: Myriad Genetics, Inc., Employee of: Myriad Genetics, Inc., Eric Sasso Shareholder of: Myriad Genetics, Inc., Employee of: Myriad Autoimmune DOI: 10.1136/annrheumdis-2021-eular.1467

\section{POS0455 EFFECT OF ANTI-Ro/SSA ANTIBODIES FOR TREATMENT RESPONSE TO METHOTREXATE IN RHEUMATOID ARTHRITIS: A RETROSPECTIVE MULTICENTER OBSERVATIONAL STUDY}

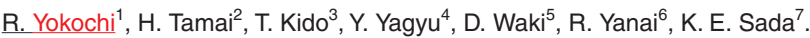
${ }^{1}$ Teikyo University Chiba Medical Center, Division of Hematology and Rheumatology, Ichihara, Japan; ${ }^{2}$ Keio University School of Medicine, Division of Rheumatology Department of Internal Medicine, Tokyo, Japan; ${ }^{3}$ Toyama University Hospital, The First Department of Internal Medicine, Toyama, Japan; ${ }^{4}$ Tokyo Kyōsai Hospital, Department of Internal Medicine, Tokyo, Japan; ${ }^{5}$ Kurashiki Central Hospital, Department of Endocrinology and Rheumatology, Kurashiki, Japan; ${ }^{6}$ Showa University Hospital, Division of Rheumatology, Tokyo, Japan; ${ }^{7}$ Kochi Medical School, Kochi University, Department of Clinical Epidemiology, Kochi, Japan

Background: Several previous observational studies have suggested that patients with anti-Ro/SSA antibody-positive rheumatoid arthritis (RA) may respond poorly to treatment, including tumor necrosis factor inhibitors ${ }^{1}$. However, its influence on methotrexate (MTX) treatment, which is the anchor drug of treatto-target strategy in RA treatment, remains unclear.

Objectives: We compared the clinical response to MTX in both anti-Ro/SSA antibody-positive and -negative patients with MTX-naiive RA and investigated the reasons for the difference in response.

Methods: We recruited 210 consecutive patients with RA who were newly started on MTX in this retrospective cohort study. The effect of the presence of anti-Ro/SSA antibodies on achieving low disease activity (LDA) of DAS28-CRP at six months after initiating MTX was investigated by using logistic regression analysis. CDAI, SDAI, concomitant using DMARDs and painkillers, patient's and evaluator's VAS, tender joint counts, and swollen joint counts at six months were also compared between the anti-Ro/SSA-positive patients and -negative patients. Missing data were imputed by using multiple imputations before multivariate analysis.

Results: 32 anti-Ro/SSA antibody-positive patients and 178 anti-Ro/SSA antibody-negative patients were included. The rate of achieving DAS28-LDA at six months was significantly lower in the anti-Ro/SSA antibody-positive patients than those in the anti-Ro/SSA antibody-negative patients $(56.2 \%$ versus $75.8 \%$, $P=0.03)$. in the logistic regression analysis, the presence of anti-Ro/SSA antibodies was an independent negative predictor for achieving DAS-28-LDA at six months (OR:0.431, 95\%Cl: 0.190-0.978, $P=0.044$ ) (Table1). Anti-Ro/SSA antibody-positive patients had significantly higher patient's VAS at six months (median [IQR]: 22 [15-41] vs 19 [5-30], $P=0.038)$, and prescribed NSAIDs (37.5\% vs $18.0 \%, P=0.018)$. CDAI and SDAl after six months were not significantly different between the group.

Conclusion: The presence of anti-Ro/SSA antibodies might be one of the predictive factors for the insufficient response to treat to target strategy in RA treatment. Residual pain was suspected as one of the mechanisms contributing to the lesser clinical response of MTX in anti-Ro antibody-positive RA.

REFERENCES:

[1] Ran Matsudaira wt al. J Rheumatol 2011;38(11):2346-54

Table 1. Logistic regression analysis for the rate of achieving DAS28 low disease activity at six months.

\begin{tabular}{lccc}
\hline Risk factor & Odds ratio & $95 \% \mathrm{Cl}$ & $P$ value \\
\hline Age at onset & 0.993 & $0.968-1.018$ & 0.586 \\
Sex (woman) & 0.643 & $0.300-1.384$ & 0.258 \\
RF-positive & 1.962 & $0.853-4.511$ & 0.112 \\
ACPA-positive & 0.552 & $0.225-1.351$ & 0.192 \\
Anti-Ro/SSA antibody-positive & 0.431 & $0.190-0.978$ & 0.044
\end{tabular}

Disclosure of Interests: None declared

DOI: 10.1136/annrheumdis-2021-eular.1485

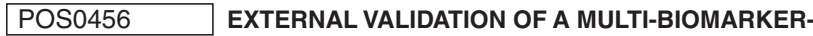 BASED CARDIOVASCULAR DISEASE RISK PREDICTION SCORE FOR RHEUMATOID ARTHRITIS PATIENTS}

J. Curtis $^{1}$, E. Sasso ${ }^{2}$, E. Hitraya ${ }^{2}$, C. Chin ${ }^{2}$, R. Bamford ${ }^{2}$, R. Ben-Shachar ${ }^{3}$, A. Gutin ${ }^{4}$, D. Flake ${ }^{4}$, B. Mabey ${ }^{4}$, J. Lanchbury ${ }^{4} .{ }^{1}$ University of Alabama at Birmingham, Immunology \& Rheumatology, Birmingham, United States of America; ${ }^{2}$ Myriad Autoimmune, $R \& D$ Medical, South San Francisco, United States of America; ${ }^{3}$ Myriad Genetics, Inc., Clinical Development, Salt Lake City, United States of America; ${ }^{4}$ Myriad Genetics, Inc., MMM Informatics, Salt Lake City, United States of America

Background: A novel score for predicting 3-year risk for CVD events in RA patients combines age, four traditional CVD risk factors (diabetes, hypertension, smoking, history of high-risk CVD event), a personalized assessment of RA-related inflammation based on the multi-biomarker disease activity (MBDA) score and, individually, 3 of its 12 biomarkers, TNF-R1, MMP-3 and leptin (log-transformed). This score was developed and internally validated using patient data from the Medicare database.

Objectives: The purpose of this analysis was to externally validate the MBDAbased CVD risk prediction score in a younger cohort from the Symphony claims database.

Methods: A cohort of patients greater than or equal to 18 years old with RA diagnosis from a rheumatologist and evidence of an RA-specific treatment, excluding patients with malignancy, past myocardial infarction (MI) or stroke, was created by a third party (Symphony) by matching medical and pharmaceutical claims and linking them to MBDA scores from a database of tests done for routine care. Medicare patients were excluded to avoid overlap with the internal validation cohort. Only the first MBDA test was used for each patient. The study endpoint was time from MBDA testing to first CVD event within a 3-year time horizon. CVD event was defined as MI or stroke, based on ICD-9 or ICD-10 diagnosis codes in hospital claims. Analyses focused on relative risk, not absolute risk, because CVD event data in Symphony may be incomplete. A univariate Cox proportiona hazards regression model was fit with the MBDA-based CVD risk score as the sole predictor of time to CVD event to obtain a hazard ratio (HR) estimate $(95 \%$ $\mathrm{Cl}$ ) and $p$-values from a likelihood ratio test (LRT). Sensitivity analyses determined HR for patient subgroups, with $p$-values determined for the interaction between subgroups and the MBDA-based CVD risk score. Using a multivariate Cox proportional hazard regression model, the MBDA-based CVD risk score was compared to a simpler model that included only age, sex, diabetes, hypertension, history of other CVD, smoking and CRP (log-transformed) for ability to predict time to a CVD event.

Results: 48,868 patients with 337 CVD events met eligibility criteria and had linked biomarker data. Mean age was 54.4 years. $81.7 \%$ were female (Table 1 ). 